\author{
Military Technical \\ College \\ Cairo, Egypt
}

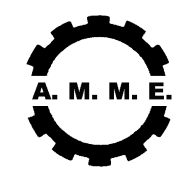

$12^{\text {th }}$ International Conference on Applied Mechanics and

Mechanical Engineering (AMME)

\title{
FAULT MONITORIMG IN COMPLEX DYNAMIC SYSTEM BY UTILIZATION STATISTICAL ANALYSIS TECHNIQUE
}

\author{
Gomaa*F.R.
}

\begin{abstract}
Vibration monitoring is widely accepted for structural defects in rotating machinery components using traditional diagnostic analysis to match spectral line with known defect frequencies that are characteristics of the affected machinery components. The present work highlights the accurate fault detect, higher order spectrum analysis ( HOSA) in rotating machinery and focus on the rare failure due nonlinearity based on compound mode which cannot appear in traditional diagnostics. And compare the results of the traditional linear spectra analysis in the case of fault monitoring of experimental tests carried out on eccentric cam in a specially designed cam dynamic test fixture. The test performed on the complex system, the cam is excited by impact hammer.

An accelerometer is used to measure the acceleration response for different manufacturing routes of cams. The ambient excitation and the system response are used to compute a number of quantities for the system under two operating conditions of surface finish. In particular the power spectrum density (PSD), the frequency response function ( FRF), the second order coherence function and the third order system bicoherence are measured and calculated. The measurements are compared and the results are discussed. The results shows that the nonlinearity due to compound mode cannot appear in linear spectra analysis. The results shows also that the traditional linear spectra analysis is of limited value in monitoring faults.
\end{abstract}

\section{KEY WORDS}

Vibration monitoring, Feature selection of damage, Detect nonlinear behavior and high order spectrum analysis .

* Assistant professor, Department of Production Engineering and Mechanical Design, Faculty of engineering, Shebin El-kom, EGYPT. 


\section{NOMENCLATURE}

HOSA High order spectral analysis technique called bispectral analysis $B\left(f_{1}, f_{2}\right)$ : Is third order spectrum can be viewed as decomposition of third moment

DFT Discrete Fourier transform $X(f)$

$X(f) \quad$ DFT of $\{X(n)\}$

$X^{*} \quad$ Indicates the complex conjugate

$O_{3 x}(i, j) \quad$ Is the third order cumulant

2DF Two degree of freedom

$\mathrm{g} \quad$ Is a dividing segment

$b^{2}\left(f_{1}, f_{2}\right) \quad$ Is a normalization form of bispectrum called bicoherence

$\mathrm{R}(\mathrm{a}) \quad$ Surface roughness of the machined cam.

PSD

Power spectrum density

FRF

Frequency response function 


\section{INTRODUCTION}

Recently, industry is showing an increasing interest in techniques aiming at the accurate fault detection in structure such as rotating machinery and in civil, aerospace. The process of implementing damage detection is referred to as structural health monitoring (SHM). Here damage is defined as changes to the material and/or geometric properties of these systems, including change to the boundary conditions and system connectivity, which adversely affected the systems performance The (SHM) process involves the observation of a system over time using periodically sample dynamic response measurements for an array of sensors, the extraction of damage-sensitivity features from these measurements, and statistical analysis of these feature to determine the current state of system health. Applying a statistical pattern recognition approach to this problem involves the following four steps,[3,4,5] (1)Operation evaluation (2) Data acquisition and cleansing (3) Feature selection and data compression (4)Statistical model development. The discussion herein focuses on feature selection for damage detection Ref $[1,2]$ summarized many feature that have been proposed for vibration -based damage detection. The majority of feature describes in this reviews are based linear time invariant, modal analysis concepts. However many damage scenarios causes the structure to respond in a nonlinear and non-stationary. Examples of such damage are the formation of cracks that subsequently open and close under normal operations and the loosening of bolted joints. Recent work at NASA Ames Research center has demonstrated that the statistical vibration in baseline (healthy)

Data as well as faulty data must be accounted for to assure accurate anomaly detection in aircraft vibration monitoring system ( Tumer and Huff, [6],[7] )

Daniel A.McAdams , Irem Y.Tumer [8] (2002) focused on global measure of vibration level as area under power spectral average density plot in frequency domain. The goal of his work was to represent all potential failure mode/defect types properties, he depends on overall level. Several technique to identify damage from vibration data have been proposed, they advanced signal processing such as wavelet analysis Ref [9] (2003) and it is suitable for periodic impulse detection. The present paper focus on the rare failure due to nonlinearity based on compound mode which can not appear in power spectrum average density (PSD) By introducing a technique for implementing ( HOSA ) for use in complex system monitoring applications and illustrate how statistical analysis detection in bicoherence in follower response effectively detect nonlinearity and isolate signal noise without depend on reference data and compared to second order spectrum analysis The techniques is applied on complex dynamic system to detect surface roughness. Using high order spectral analysis and traditional linear spectral are measured and compared.

\section{THEORETICAL STATISTICS ANALYSIS OF VIBRATION SIGNATURE USING HIGH ORDER SPECTRAL ANALYSIS}

From signal processing, the behavior perspective running cam system has complex nature The spectrum can be considered as a mixture of deterministic effect from cam contact to the follower in conjunction with random signal generating from cam profile error and other geometric parameters The weaker signals, contains more significant information relating cam surface error, environmental condition and / or experimental noise. The location of an accelerometer on the follower arm under investigation is 
convenient for picking up random vibration in the structure (which contains a mixture of data of interest and often unwanted information).

In such case one critism of the traditional linear spectra analysis is a limited value in vibration analysis where nonlinearities are involved $[8,10]$.

In studying the follower response due to nonlinearities and noise in experimental work, the higher order (HOSA) have efficient tools for the of vibration at which the generation and interaction on non-linear resonance modes are of major concern. In [11,12 ] The (HOSE) technique was used as nonlinearity detector .Rivola ,A, and others [ 10 ] stated that a beam with fatigue cracks has a bilinear nature, that is the system has a non-symmetric and nonlinearity nature Therefore the bispectrum should expected to be sensitive the fault signals since the bispectrum is defined as the frequency decomposition of the third order, or third order covariane function of a signal. Hence the bispectrum provides information regarding such signal features as phase coherence which are absent in the second-order power spectrum (Autospectrum).

The result is that bispectral investigates the statistical relationship between the spectra components where the correlation between these spectra components are given in question [10] such as coupling and quadratic mode coupling or combinational resonance modes and the form

$$
\mathrm{B}_{x}\left(f_{1}, f_{2}\right)=E\left[x\left(f_{1}\right) x\left(f_{2}\right) x^{*}\left(f_{1}+f_{2}\right)\right]
$$

$f_{1}, f_{2} \quad$ : is a function of two independent frequency

$\mathrm{x}(\mathrm{f}) \quad$ : is a discrete of frequency transformation of $\{\mathrm{x}(\mathrm{n})\}$

$x^{*} \quad$ : indicates the complex conjugate

$\mathrm{E}[\mathrm{]}$ : denotes the statistical expected value.

The autocorrelation function of $\{x(n)\}$ is

$$
R_{x x}(i)=E\{x(n) x(n+i)\}
$$

and the power spectrum of $\{x(n)\}$ is defined as Fourier Transform of autocorrelation. In (HOSA), The higher order cumulants are defined as follows

$$
\begin{aligned}
& O_{3 x}(i, j)=E[X(n) X(n+i) X(n+j)] \\
& O_{3 x}(i, j) \quad \text { is the third order cumulant }
\end{aligned}
$$

Similarly, the bispectrum is defined as 2D.F of the third order cumulants.

The estimating of bispectrm can be represented as $O_{3 x}(i, j)$

$$
B\left(f_{1}, f_{2}\right)=E\left[X\left(f_{1}\right) X\left(f_{2}\right) X *\left(f_{1}+f_{2}\right)\right]
$$

For monitoring fault signals using Bispectrum, the estimator of the higher order is zero according to the third order cumulant of zero mean Gaussian process in normal situation for orders greater than two

$$
O_{3 x}(i, j)=0
$$




$$
B\left(f_{1}, f_{2}\right)=0
$$

In normal situation the vibration signal is close to Gaussian. When damage happens, the signal often deviates from Gaussian. So the stationary state of machine can be observed the bispectrum to be zero or not .In practice, the normalization bispectrum is referred to be as the bicoherence which is defined by [17].

$$
b^{2}\left(f_{1}, f_{2}\right)=\frac{\left|B_{X}\left(f_{1}, f_{2}\right)\right|^{2}}{E\left[\left|X\left(f_{1}\right) X\left(f_{2}\right)\right|^{2}\right] E\left[\left|X\left(f_{1}+f_{2}\right)\right|^{2}\right]}
$$

One advantage of the normalization is to make the variance approximately flat across all frequencies in the case of phase coupling of the nonlinear system. For random signals the bicoherence is a measure of signal skew ness. The main feature of bicoherence is that it has binary form (to vary between 0 and 1) [11,12] and quantitatively measures the fraction of the power of the signal due to the quadratic interaction between frequency components [15 ] Here one indicates that all energy at $f_{1}+f_{2}$ is a result from coupling of components at $f_{1}$ and $f_{2}$. Therefore, this function can be used to detect the presence of harmonic. One advantage of the bicoherence is to require a single measurement instead to the coherence required to input and output signals. The bicoherence can be calculated as discrete system For each segment equations (1) and (7) can be computed by using the discrete Fourier Transform and is followed by obtaining the statistical estimate by averaging over all segments. The bicoherence can be calculated as the form

$$
b^{2}\left(f_{1}, f_{2}\right)=\frac{\left|\sum_{i=1}^{g} X_{i}\left(f_{1}\right) X_{i}\left(f_{2}\right) X_{i}^{*}\left(f_{1}+f_{2}\right)\right|^{2}}{\sum_{i=1}^{g}\left|X_{i}\left(f_{1}\right) X_{I}\left(f_{2}\right)\right|^{2} \sum_{i=1}^{g}\left|X_{i}\left(f_{1}+f_{2}\right)\right|^{2}}
$$

where $\mathrm{g}$ is a dividing segment.

Moreover, since the signal encountered in practice are often contaminated by Gaussian measurement noise, of which second order auto correlation measure are theoretically insensitive. The bispectrum are potentially powerful tools for analysis real-life signals by using the superposition theorem where the cumulant signal is the sum of cumulant statistically independent signals. According to the theorem of superposition if the signal is added by Gaussian noise, the effect of noise can be suppressed in higher order cumulant domain.Bispectrum can be use in harmonic detection where symmetry shark peaks as the quadratic harmonic exists [13].It is difficult to detect this harmonic in power spectrum averaging.

\section{EXPERIMENTAL TESTING}

To verify the proposed method of analysis, an inexpensive cam dynamic test fixture is designed and built with two types of machine eccentric cam as shown in Fig 1a,b. For monitoring acceleration response of the follower arm, the cam as in Fig.1b running at constant velocity under ambient excitation (Random excitation). The data consists of time and frequency history throw one accelerometer mounted directly above the flat follower arm Fig. 2 . 
The acceleration is fed to conditioning amplifier and subjected to Fast Fourier Transform (F.F.T) analyzer. The signals are recorded and transformed to frequency domain. All signals are stored in frequency domain and time domain using filtering and hanning window.

\subsection{Auto Spectrum Measurement}

Using ambient excitation, the power spectrum average of the acceleration response are measured during a series of cases ( two different degrees of surface roughness, turning and grinding manufacturing for the cam ).

The surface error is measured using teleserf as shown in Fig 3a,b. And table1. Fig 4a,b.shows the auto spectrum density (PSD) of the follower arm. The examined frequency range limited to the frequency range $(0-100 \mathrm{~Hz})$ Because most of the changes due to the addition to surface error to the cam profile are observed in the lower frequency range $(0-50 \mathrm{~Hz})$ as shown in

Fig.5a,b we select the total power in this range. The (PSD)are computed by average 100 segments ( Hanning window is employed Fig. a,,b shows a zoomed in portion of lower frequency range where the difference due to the two signals can be seen clearly. In general, the addition of the profile and surface errors introduces frequencies in noise range, as well as increasing the overall power level, and power spectrum is of limited value in studying the behavior of nonlinearity systems

\subsection{FRF Measurement .}

In order to detect the nonlinearity introduced by damage and/or environmental condition, The (FRF) and second order coherence function have been measured and estimated, in two cases as in Figs.6,7. the processing parameters are the same as in the case of power spectrum, that is the frequency resolution is identical $(2.5 \mathrm{~Hz})$.

Fig.6,7 shows the FRF of the system for linear spectra analysis and the coherence function, the motor is shut down and the cam follower system is exited by impact hammer, the system response is measured by means of an accelerometer mounted directly on the flat follower as in Fig. 2

\subsection{HOSA Technique}

In this paper we first focus on the fault detection on residual signal (where the main frequency components have been removed in PSD).

According this the higher order spectral analysis ( HOSA )., can used as nonlinearity detectors $[10,12]$. Bispectrum of the vibration signals are computed using Matlab tool box. When a non -linear system is excited by random input, it usually generates response frequencies are multiple and / or combination of structural resonance frequencies, sometimes it generates sub-harmonic frequencies. The HOSA of the follower acceleration response are then considered in order to look for the generation of non-linear modes. The normalized biocherence is computed from the total data length with DFT as shown in Figs.8,10.for Turned and grinded cam respectively. 


\section{RESULTS AND DISCUSSION}

\subsection{Analysis OF Cam Follower Vibration Signature Using (PSD)}

The vibration signature needs to be under stand to decide how dose response vibration (vibration response distribution ) related to parameters of specific system being monitored .To apply the possible set of features that will be used to monitor system performance and indicate the occurrence of errors in profile that introduces random input excitations and generates response at frequencies that are multiples and / or combinations of spectral resonance frequencies (sometimes it generates sub-harmonics frequencies). A sample of simulated cam follower vibration response for cam machined with turning and grinding respectively. The frequency content of these signals is shown in Fig. 4a,b ,the examined frequency ranges is $(0-100 \mathrm{~Hz})$, the ( PSD ) of the system accelerometer response are then considered in order to look for generation of non-linear modes. The difference in PSD level due to additional profile and surface error that appear in lower frequency. The second plot Fig $5 a, b$ shows zoomed-in portion of lower frequency $(0-50 \mathrm{~Hz})$, where the difference due to two errors in roughness causes only small change in auto spectrum amplitude shown in Fig. However they do not provide much information about the nature of the system. That they do not tell any thing about the generation of combinational components due to nonlinearities. This confirm that ( PSD) is of limited value in studying the behavior of nonlinear system or residual signals.

Many possibilities exist for selecting feature set for purpose of monitoring change in vibration signature [ 7,14 ] .Daniel A., Irem Turmer [8] focused on global measure of vibration level as area under PSD plot in frequency domain (equivalent to variance in time domain ). The goal of their work was to represent all potential failure modes/defects types properties and this method need to reference data in integer system , and give overall measure of system non linearity, The present paper focuses on the rare failure due to non linearity based on compound mode which can not appear in ( PSD ).

\subsection{Analysis OF Cam Follower Vibration Signature Using (FRF)}

In order to detect nonlinearity introduced by error, the frequency response function (FRF ) and second order coherence function have been measured using the same frequency resolution as in (PSD ). The comparison between Fig .6 and Fig. 7 shows that FRF, sensible to peak due to nonlinear behavior of the cam follower with the surface error. In particular, there is a clear non-linear peak at frequency $(9.750 \mathrm{~Hz})$ in addition two small peaks at $5 \mathrm{~Hz}$ and $40 \mathrm{~Hz}$ in turning Fig. 6 shows FRF for grinding cam there is also clear non-linear peak at frequency $5 \mathrm{~Hz}$. At frequencies of the combinational components the system response is not correlated to input of the system. Consequently the coherence is less than unity at those frequencies as shown in the same Figs 6,7, this is particular evident at frequency ( $10 \mathrm{~Hz}$ ) where nonlinear effect takes place for error in surface of the cam. Fig .6,7 show the coherence drop for the first and the second cams. We can conclude that ( FRF, coherence) give a clear information of nonlinear but the comparison between integrated system and fault system is needed. FRF needs input and output measurements and two accelerometers, in contrast on HOSA needs one accelerometer for ambient excitation. 


\subsection{Bicoherence (HOSA) High Order Spectrum Analysis}

Because of the goal of this paper to detect rare failure due to non-linearity in complex system without the need to the integer system. (HOS) ( higher order spectrum is used as alternative procedure. The acceleration signal measurements are used to compute the bicoherence.

Figs.9,10. plot the bicoherence generated from the vibration of the cam follower for the two machining conditions ( turning and grinding) it clearly appears that the bicoherence is very sensitive to surface error. The bicoherence rises in case of turning surface as in Fig.8,10.

and table 1.

Thus showing a significant coupling between frequencies due to system nonlinearities. The bicoherence measures the normalization degree of statistical dependency without reference to spectral amplitude, it detects the non-linear interactions in those cases where the resultant spectral components is hardly noticeable, as in the PSD of Fig. 5a,b The bicoherence links a sum of frequencies each near $10 \mathrm{~Hz}$ and $19 \mathrm{~Hz}$,to response at about $29 \mathrm{~Hz}$. This results is clearly displayed in Figs. 8,10 ..

there is significant bicoherence at the frequencies pairs $(\approx 10, \approx 19 \mathrm{~Hz})$, this phenomena indicates a coupling between frequencies component at the triplet $(10,19,29 \mathrm{~Hz})$ when the nonlinearity arises, the system response shows several components having frequencies which is multiple of natural frequencies of the linear system.

This is evident in the bicoherence but comparing in PSD a week components at frequency $29 \mathrm{~Hz}$. As in Fig 5a,b, Examination of PDS shows two peaks at $19 \mathrm{~Hz}$ as well as $10 \mathrm{~Hz}$ Fig 5a,b However, this plot gives no indications of the coupling between these frequencies that is evident in bicoherence.

Table1. Feature of bicoherene response

\begin{tabular}{|l|c|c|c|}
\hline Machining type & $\begin{array}{c}\text { Roughness } \\
\text { using telesurfe }\end{array}$ & Max bichorence & $\begin{array}{c}\text { Frequency } \\
\text { components }\end{array}$ \\
\hline Grinding & $0.01 \mu \mathrm{m}$ & 0.382 & $(10,19,29) \mathrm{Hz}$. \\
Turning & $0.054 \mu \mathrm{m}$ & 0.596 & $(10,19,29) \mathrm{Hz}$. \\
\hline
\end{tabular}

By observing the bicoherence's contour plot Figs.9,11., it is possible to understand how the system resonance interacts causing the birth of nonlinear component in the case of error $0.054 \mu \mathrm{m}$ the highest bicoherence is located at the frequency pair $(10,19 \mathrm{~Hz})$ This indicates a coupling between frequency component at $(10,19,29 \mathrm{~Hz})$ that indicates the two modes at frequency $10 \mathrm{~Hz}, 19 \mathrm{~Hz}$ interact and lead to the compound resonance at $29 \mathrm{~Hz}$. Fig. 9 shows other coupling between spectral component are not visible in the FRF of Fig.5a. In conclusion, the bispectral analysis provide additional insight for identifying the dynamic response of system's nonlinearity . 


\section{CONCLUSION}

1- The analysis using HOSA (The bicoherence) has the advantage of measuring the normalized degree of statistical dependency without reference to spectral amplitude, compared to second order spectrum analysis, it detects the non linear interaction even in

those cases where the resultant component is hardly noticeable as in power spectrum Fig. 5a,b

2-The measurements are evaluated from output signal only to determine whether and how

manufacturing variations propagate through the system in contrast to FRF measurements, so

they can be represented in the signal modeling for machine health monitoring system.

3-The test analyzed in this study are performed in a laboratory with constant speed, there for, had

minimal environmental variability associated with them. For field application by changing

environmental and operating conditions, statistical pattern recognition to features can be derived

from bispectral analysis.

4-The bicoherence measure has been adopted as a tool for detecting profile errors in complex

system. .

5-The bicoherence feature can be utilized in the future to implement an automatic fault

detection procedure because it depends on ambient excitation. 


\section{REFERENCES}

[1] Doubling ,S.w,Farran C.R., Prime, M.B and Sheviz D.w Damage identification and health monitoring of structural and mechanical systems form changes in their vibration characteristics: literature Review.LA-13070-MS1996. Los Alamos national Laboratory.

[2] Doubling ,S.w,Farran C.R., Prime, M.B and Sheviz D.w 1998 A review of damage identification methods that examine change in dynamic properties shock and vibration Dig. 30

[3] Farrar C.R , Duffey T.A, Doebling S.Wand Nix DA (2000) A statistical pattern recognition for vibration based on structural health monitoring proc. $2^{\text {nd }}$ In workshop on structural health monitoring(Stanford,cn September. 8-10 2000 pp 764-773.

[4] Fugate ML,Sohn H and Farran CR 2000 vibration based damage detection using statistical process control Mech.syst .signal process at press

[5] Sohn H C2arneck JJand Farrar C.R 2000 Structural health monitoring using statistical process control J.Eng. Mech,ASCE at press

[6] Tumer, I and Huff,E.(2000) .Evaluating manufacturing and assembly errors in rotating machinery to enhance component performance. In ASME Design for manufacturing conference, volume DETC 2000/DFM-14006, Baltmore MD.

[7] Tumer, I and Huff., E. 2001. Using triaxial accelerometer data for vibration monitoring of helicopter gearboxes. In ASME Mechanical vibration and noise conference, volume DETC 2001/VIB-21755 Pittsburgh,PA.

[8] Dniel A ,McAdams, PhD and Irem,Y.Tumer,phD Towards failure modeling in complex dynamic systems Impact of design and manufacturing variations proceedings of DETC,(2002 ASME Design Engineering Technical conferences September 29- October 2, (2002) Monterial, Quebec Canada.

[9] J. lin and M.J. Zuo "Gearbox fault Diagnosis using adaptive wavelet filter. Mechanical system and signal processing (2003) 17 (6) ,1259-1269."

[10] Rivola,A., and white P.R., Bispectral Analysis of The bilinear oscillator with application to the Detection Fatigue cracks. Journal of sound and vibration 1998,216(5),889-910.

[11] - Fackrell,J.W.A., Mclaughlin,S., " TheHigher Order Statistics of Speech Signals" Proceedings of the IEE Colloquium on Techniques in speech signal processing London, Digetal No.1994/138,7/1-7/6.(1994).

[12] Fackrell,J.W.A., and white P.R, Hammond,J.K.,Pinning ton,R. Parsons, A.T. "The Interpretation of the Bispectra of Vibration Signal : part 1 Theory, Mechanical Systems and Signal processing , Vol,9,No 3, 257-266-(1995).

[13] Xiaoming Gao, Ovaska s.j Analysis of second order harmonic distortion of ADCusing Bisoectrum. IEE Transactions on Instrumentation and measurements 1996, 45 (1), 50-55

[14] Smith, J.D. (1999) Gear noise and their vibration. Marcel Dekker.

[15] Collis, B. ,white P.R. and Hammond, J.K. ,Higher order spectra The Bispectrum and Trispectrum. Mechanical system and signal processing, 1998, 12(3), 375394.

[16] C.J.Li Ma, and B. Hwang (1995) Transaction of the ASME,Journal of Engineering for Industry117, 625-629. Bearing localized defect detection by bicoherence analysis of vibrations.

[17] Kim, Y. C. and powers, E. J., Digital Bispectral Analydis and its Applications to Non linear wave Interactions IEEE Transactions on plasma Science, (1979)- 7, 120-131. 


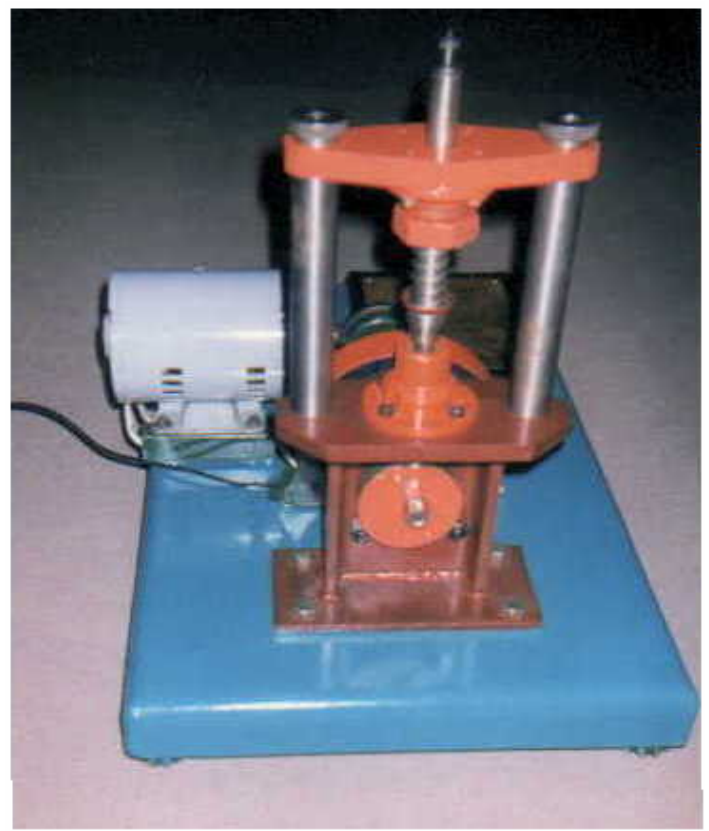

Fig. $\left(1 a_{)}\right.$: Cam dynamic test fixture

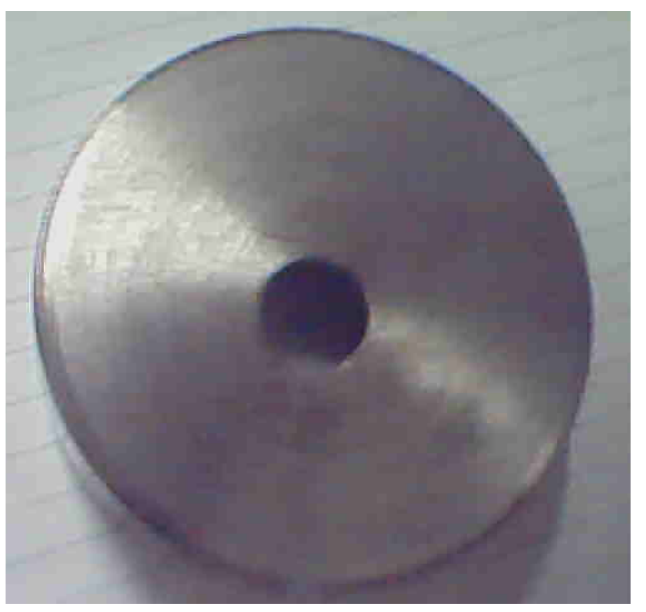

Fig.1.b Eccentric test cam 


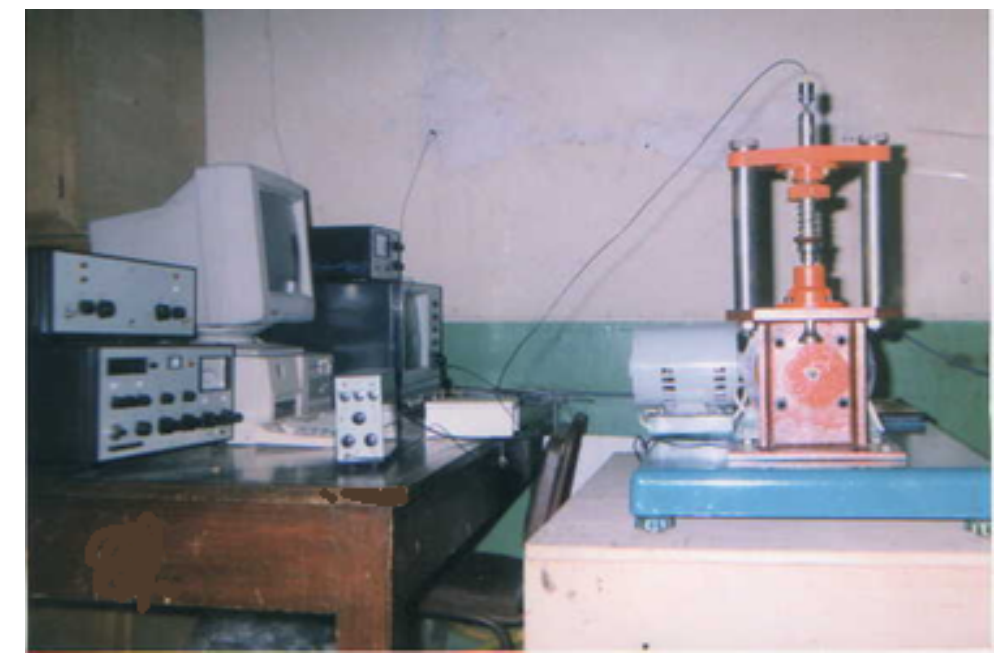

Fig.2 Setting up the measuring system

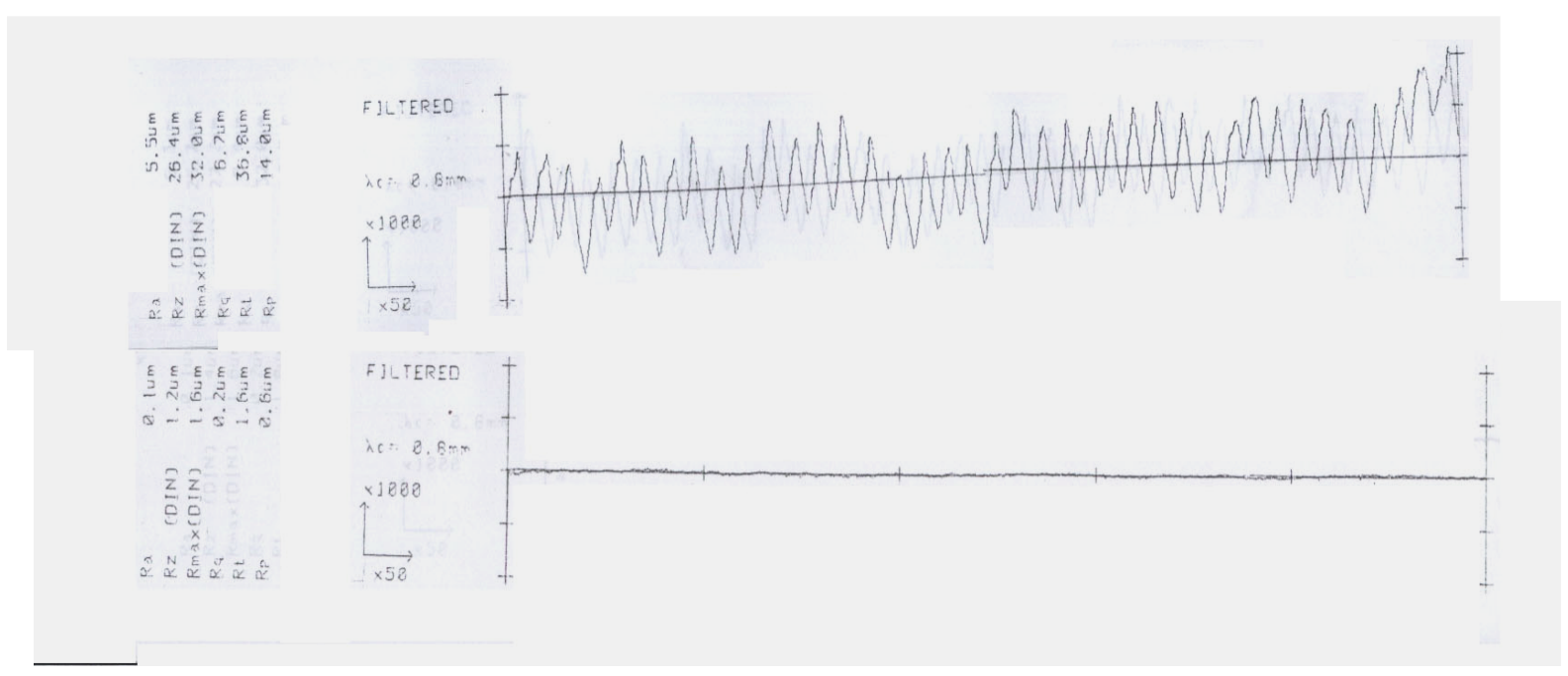

Fig.3.a,b Sample of measuring surface roughness (Ra) ( for turned and grinded cam ) 


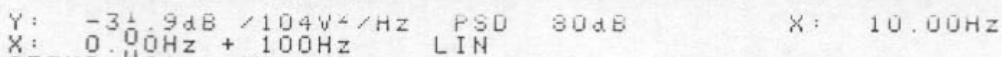

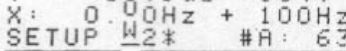

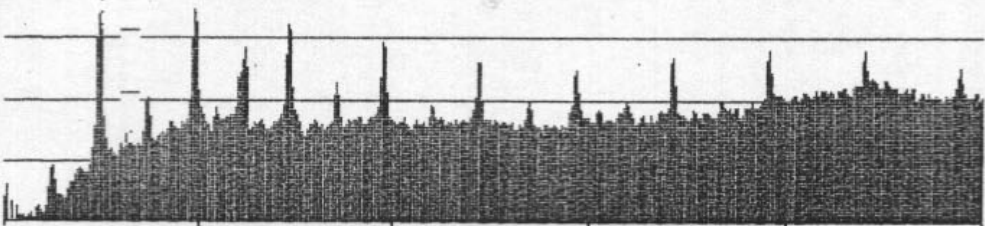

SETUP W2

MEASUREMENT: CH.E SPECTRUM AUERAGIHG
TRIGGER:

AVERAGING: LIN 100 OVERLAP: MAX

FREQ SPAN: $200 \mathrm{~Hz} \quad \triangle F: 250 \mathrm{mHz} \quad T: 4 \xi \quad 4 T: 1.95 \mathrm{~ms}$ CENTER FREQ: BASEEAND
WEIGHTIN: HAHHING

CH. A

GENERATOR

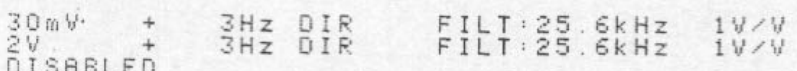

Fig. (4a): Power spectrum of the follower acceleration (Turned cam )

W1 RUTO SPEC CHE X: $0.00 \mathrm{~Hz}+100 \mathrm{~Hz}$

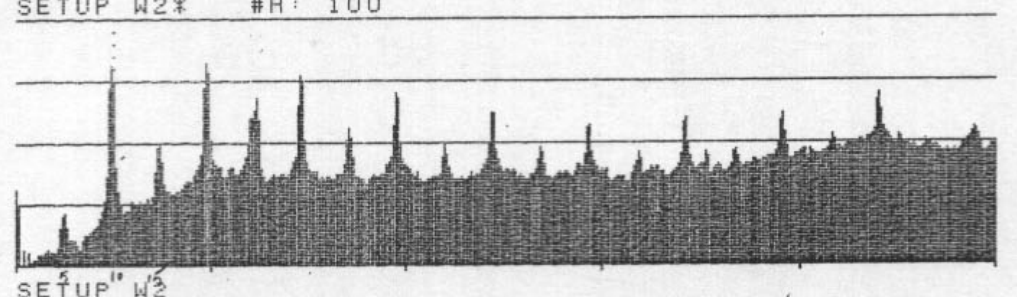

$\begin{array}{ll}\text { MEASUREMENT: } & \text { CHE SPECTRUM AUERAGING } \\ \text { TRIGGER: } & \text { FREE RUM }\end{array}$

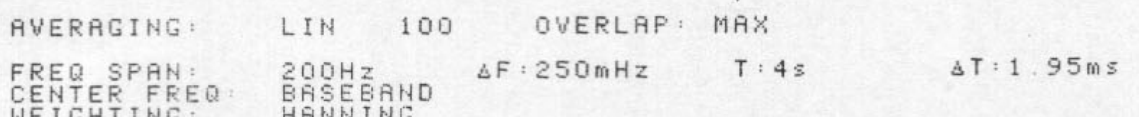
CENTER FREQ

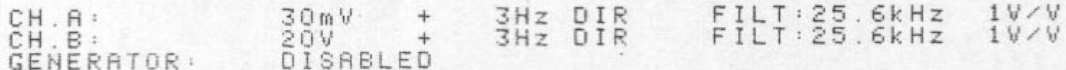

Fig (4b) : Power spectrum of the follower acceleration (Grinded cam)

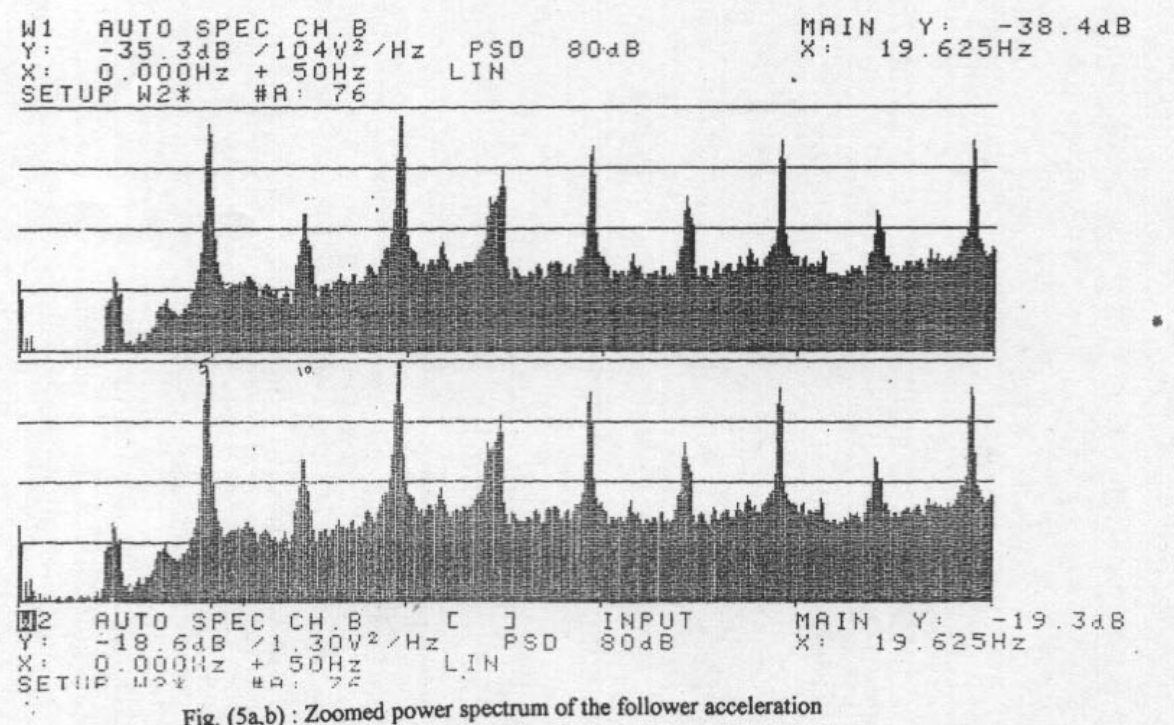




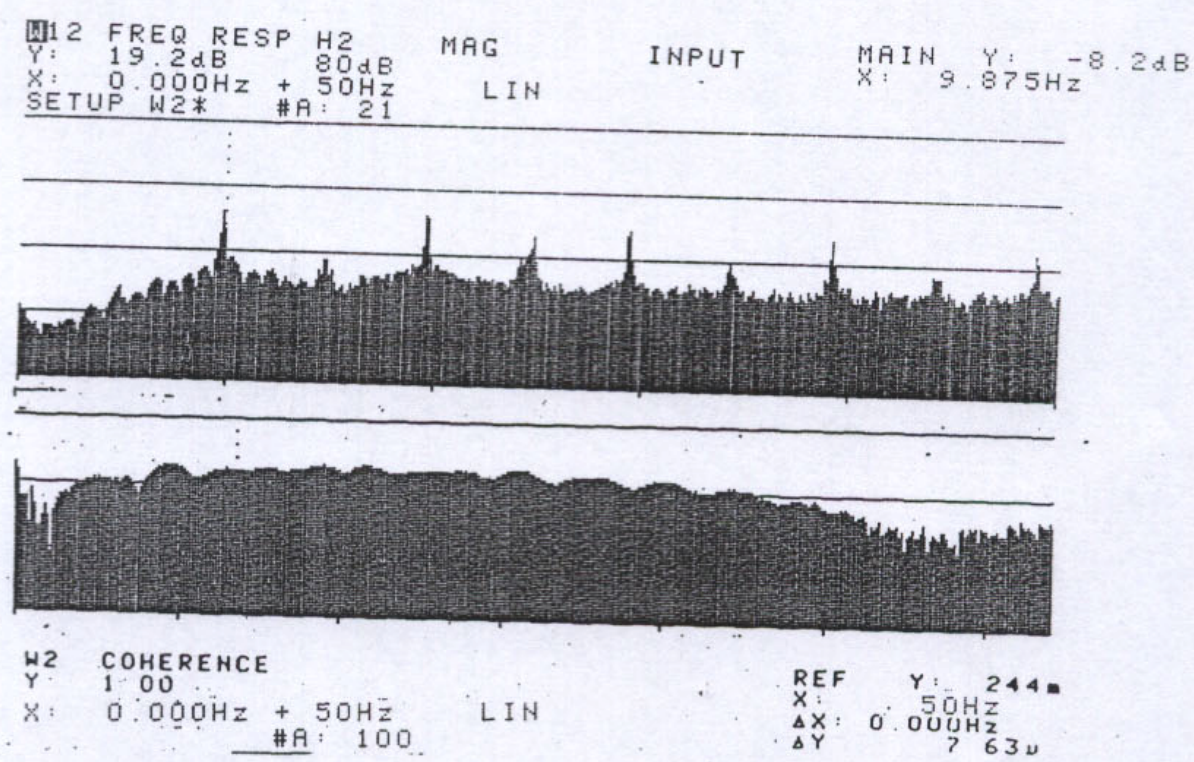

Fig. (6) : FRF between input and output of the system and second order coherence function ( Turned cam)
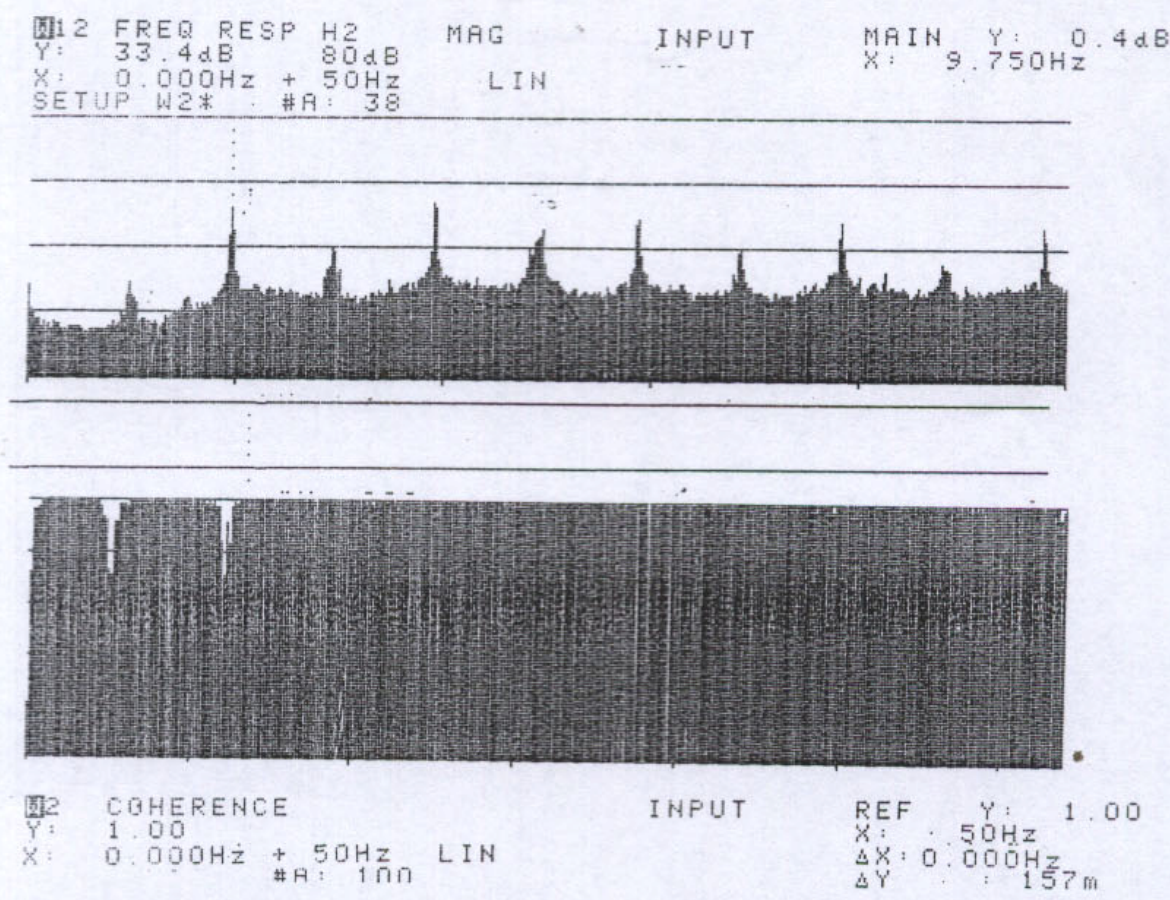

Fig. (7) : FRF between input and output of the system and second order coherence function (Grinded cam ) 


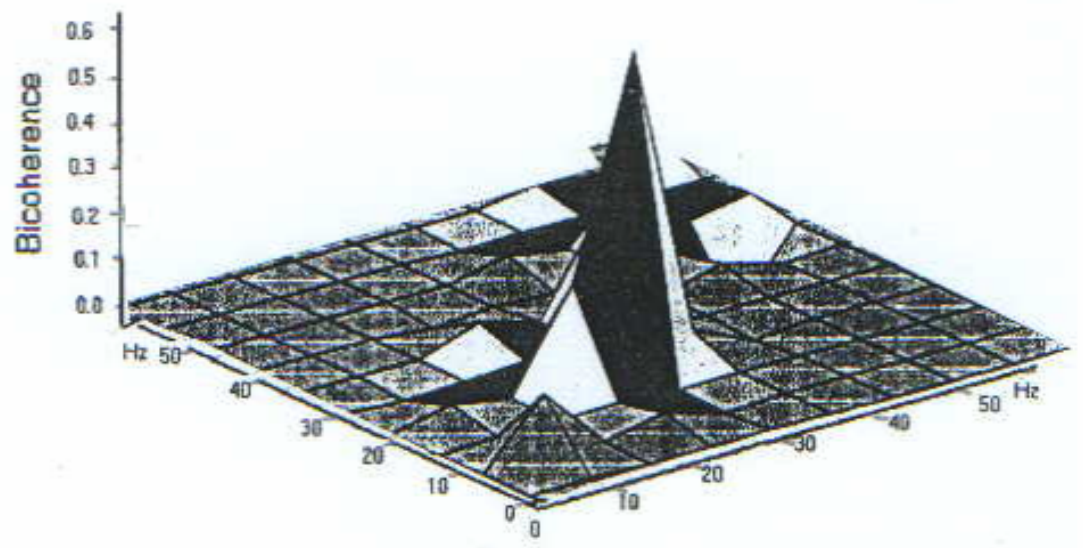

Fig. (8 ) Bicoherence of the follower acceleration (Cam roughness $=0.054 \mathrm{um}$ )

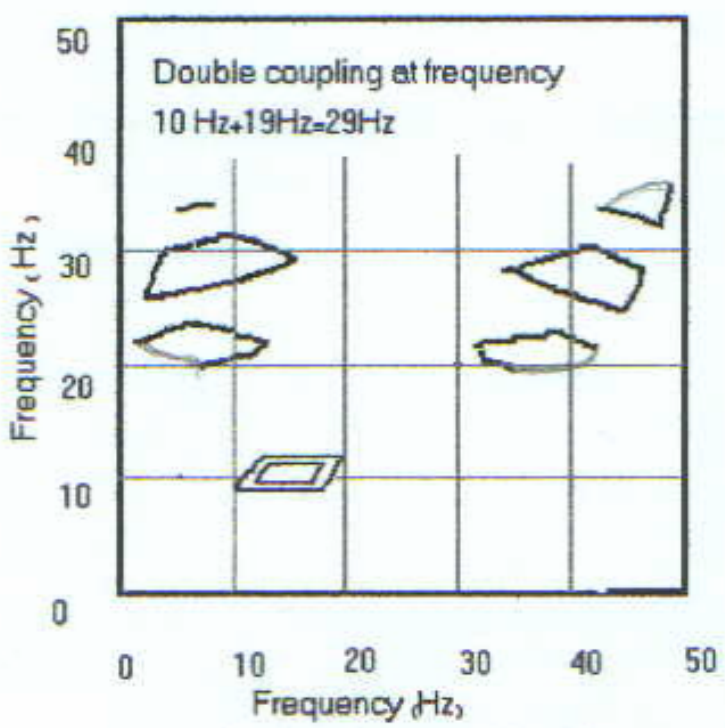

Fig. ( 9 ): Contour plot of bicoherence of the follower acceleration response, Turned cam, 


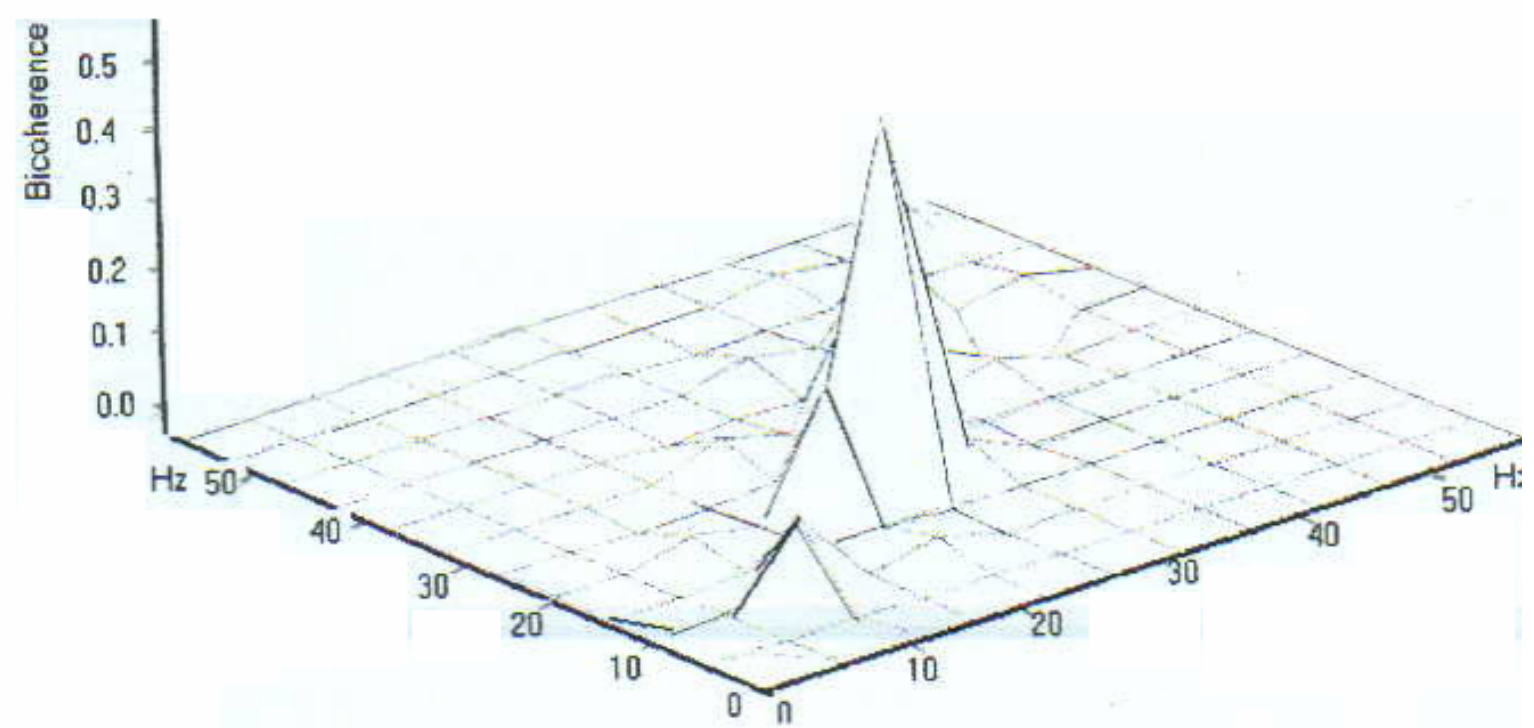

Fig. 10, Bicoherence of the follower acceleration (Cam roughness $=0.01 \mu \mathrm{m}$ )

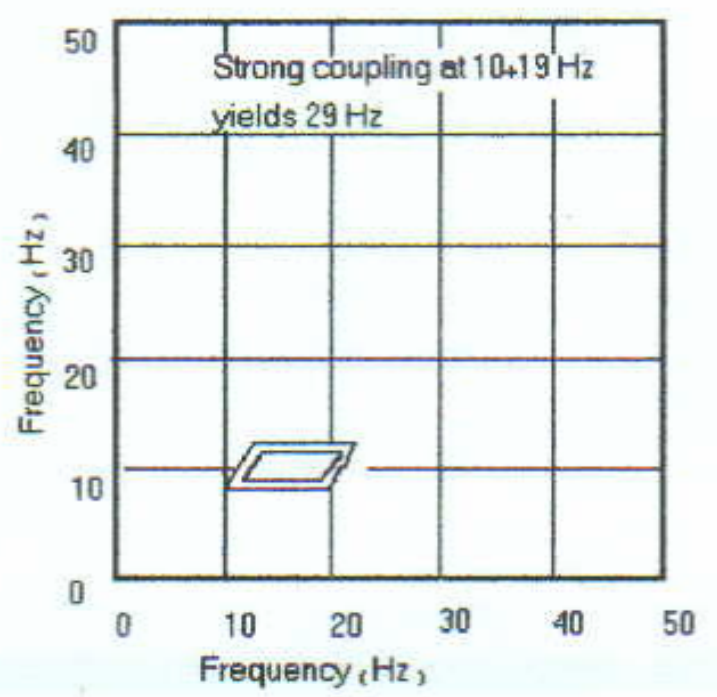

Fig. (11) Contour plot of the bicoherence of the cam follower acceleration response , Grinded cam , 